\title{
Further Developments in Dynamic Focusing
}

By J. Irwin et al.

1999 Particle Accelerator Conference, 3/29/99ã4/2/99, New York, NY, USA

Stanford Linear Accelerator Center, Stanford University, Stanford, CA 94309

Work supported by Department of Energy contract DE AC03 76SF00515. 


\title{
FURTHER DEVELOPMENTS IN DYNAMIC FOCUSING*
}

\author{
J.Irwin, D.Helm, K.Thompson, SLAC, Stanford, CA, D.Schulte, CERN, Geneva, Switzerland
}

\begin{abstract}
Dynamic focusing has been proposed[1] as a way to eliminate a conventional collimation and final focus system in linear colliders, and is a scheme that is more readily extended to colliders at several $\mathrm{TeV}$ center-of-mass energy. In this paper we examine several outstanding issues, in particular, the optimization of the lens and main beam parameters. Simulations of the lens-lens, lens-main, and main-main beam collisions using a modified version of the GUINEAPIG beam-beam code are in progress.
\end{abstract}

\section{INTRODUCTION}

Dynamic focusing has been discussed in earlier conference papers $[1,2,3]$. We continue with what we hope is a more insightful discussion of the equations controlling the lensbeam parameters, accompanied by the first particle tracking simulations. The GUINEAPIG code[4] has been modified to support very unequal bunch lengths (necessary for the lens-main collison). A full simulation begins at the entry to the lens-lens collision after the particle distribution has been shaped by the octupole modules[3], followed by simulation of the lens-lens collision, the lens-main collision and the main-main collisions. The dependence of the system on both the main-beam and lens-beam bunch length are of special interest. The main-beam bunch length can be varied to achieve maximum luminosity in the mainmain collision, while the lens-beam bunch length should be made as long as possible in order to minimize the synchrotron radiation[5] of the main beam in the lens-main collision.

\section{THE PINCH EQUATION}

The central equation in the determination of lens-beam parameters is the pinch equation[6]. For a diverging lens beam, assuming for simplicity that the main beam has a uniform longitudinal distribution, this equation has the form

$$
\frac{1}{60}\left(\frac{\sigma_{z, M}}{f_{Q}} \frac{\ell^{*}}{\beta^{*}}\right)^{2}=\frac{<s^{2}>}{\beta^{* 2}}=\left(\frac{\sigma_{f}^{2}-\sigma_{0}^{2}}{\sigma_{0}^{2}}\right) .
$$

where $s$ is the displacment of the focal point, $\beta^{*}$ is the main-beam beta function at the IP, $\ell^{*}$ is the distance between the lens-main collision and the IP, $\sigma_{z, M}$ is the mainbeam bunch length, $\sigma_{0}=\sqrt{\epsilon_{M} \beta^{*}}, \sigma_{f}$ is the final rms size of the main beam at the IP taking into account beam enlargement from the pinch effect, and $f_{Q}$ is the focal

\footnotetext{
* Work supported by Department of Energy Contract DE-AC03$76 \mathrm{SF} 00515$
}

length experienced by the lens beam as it passes through the main beam. The ratio $\ell^{*} / \beta^{*}$ equals the demagnification $\xi=\sigma_{M} / \sigma_{0}$, where $\sigma_{M}$ is the size of the main beam at the lens-main collision. Introducing the formula for the focal length $f_{Q}$, and noting the presence of factors which occur in the definition of the main-beam disruption at the IP, we may write Eq. 1 as

$$
D_{0} \frac{\gamma_{M}}{\gamma_{Q}}=\sqrt{60\left(\frac{\sigma_{f}^{2}}{\sigma_{0}^{2}}-1\right)} \cdot \xi
$$

where $D_{0}$ is the disruption parameter based on a beam size $\sigma_{0}$ at the IP. Since the actual beam size is $\sigma_{f}$, it is the disruption parameter based on this beam size which is relevant to determining the luminosity enhancement. Therefore it is better to write this equation as

$$
D_{f} \frac{\gamma_{M}}{\gamma_{Q}}=c_{p} \xi \quad \text { where } \quad c_{p} \equiv \sqrt{60 \frac{\sigma_{0}^{4}}{\sigma_{f}^{4}}\left(\frac{\sigma_{f}^{2}}{\sigma_{0}^{2}}-1\right)}
$$

As the main-beam bunch length is increased, the pinch aberration will cause the ratio $\sigma_{f} / \sigma_{0}$ to grow. $c_{p}$ has a maximum of $c_{p}=\sqrt{15}=3.87$ at $\sigma_{f} / \sigma_{0}=\sqrt{2}$, and a value $c_{p}=\sqrt{40 / 3}=3.65$ at $\sigma_{f} / \sigma_{0}=\sqrt{1.5}$. Since it is a major advantage to have a large $\gamma$-ratio and a large disruption, it will be advantageous to operate with a mainbeam bunch length that gives an apparent 50\% luminosity loss due to the lens pinch aberration, since this loss is more than compensated by the enhancement achievable from the larger disruption parameter. Simulations are underway to see how this plays out when there is no assumption on the longitudinal uniformity of the lens beam. A sensible set of parameters satisfying Eq. 3 are $D_{f}=1.2$ (giving a roundbeam enhancement $H \approx 4$ ), a $\gamma$ ratio of about 100 , and a demagnification of 33 .

\section{THE POWER-RATIO EQUATION}

We have previously shown[3] that

$$
\frac{\ell^{*}}{f_{Q}}=\frac{P_{M}}{P_{0}}=\frac{\gamma_{M} N_{M}}{\gamma_{Q} N_{Q 0}} \quad \text { where } \quad N_{Q 0}=\frac{\gamma \epsilon_{M}}{r_{e}} \xi
$$

The charge in the uniform disk of the lens beam is $\left(\sigma_{Q} / \sigma_{M}\right)^{2} N_{Q 0} \approx 3 N_{Q 0}$, where the ratio $\left(\sigma_{Q} / \sigma_{M}\right)^{2}$ is chosen so that only about $10 \%$ of the main beam tail is not properly focused. Using the $\gamma$-ratio as determined by Eq. 3 , Eq. 4 becomes

$$
\frac{\ell^{*}}{f_{Q}}=c_{p} \frac{\sigma_{f}^{2}}{\sigma_{0}^{2}} \frac{F r_{e}}{\gamma_{M}} \frac{\beta^{*}}{D_{F} H} .
$$


$F$, which has the dimension of a flux, is basically a ratio of luminosity to beam power, coming from the luminosity equation:

$$
\frac{N_{M} H}{\sigma_{f}^{2}}=4 \pi L \frac{E_{B}}{P_{B}} \equiv F .
$$

Assuming beam power is allowed to increase as the square root of the energy, $F / \gamma_{M}$ is increasing as energy to the $3 / 2$, while all other parameters in Eq. 5 tend to remain constant. Hence it is difficult to hold $\ell^{*} / f_{Q}$ constant at energies of 5 $\mathrm{TeV}$ c.m. and above. However the product $D_{f} H$ and $\beta^{*}$ can change some.

Eq. 5 may also be regarded as an equation for $f_{Q}$ :

$$
f_{Q}=\frac{1}{c_{p}} \frac{\sigma_{0}^{2}}{\sigma_{f}^{2}} \frac{\gamma_{M}}{F r_{e}} D_{f} H \xi
$$

To maintain a diverging lens-beam geometry, we require $\beta_{Q}^{*} \leq 3 f_{Q}$, so $f_{Q}$ cannot be allowed to get too small.

\section{THE LENS-LENS COLLISION}

As previously noted, the lens-lens collision can be used for self-alignment[3]. In that case

$$
\frac{\ell^{*}}{f_{Q}}=2 \sqrt{2 \frac{\gamma_{M}}{\gamma_{Q}}}+2 .
$$

For a $\gamma$ ratio of 100 , we find $\ell^{*} / f_{Q}=30.2$. Table 1 gives parameters (not yet fully optimized) for c.m. energies from $0.5 \mathrm{TeV}$ to $3 \mathrm{TeV}$ with $\ell^{*} / f_{Q} \approx 30$. In all cases, $\sigma_{Q}^{2}=R_{Q}^{2} / 2$, demagnification $\sigma_{M} / \sigma_{0}=\ell^{*} / \beta^{*} \approx 35$, $\beta^{*} / f_{Q}=0.67,\left(\sigma_{f} / \sigma_{0}\right)^{2}=1.5,\left(\sigma_{Q} / \sigma_{M}\right)^{2}=3$, $N_{Q} / N_{Q D}=1.2, f_{Q}=\sigma_{z, Q}, A \equiv \sigma_{z, M} / \beta^{*}=0.18$, $D_{f}=1.27$, and an analytic estimate of luminosity enhancement factor $H_{D}=3.67$. The main beam power $P_{B}=$ $80 \mathrm{MW}$ for $1 \mathrm{TeV}$ c.m. and scales as $\sqrt{E}$, but the luminosity $L$ departs somewhat from $E^{2}$ scaling.

\section{THE DEMAGNIFICATION PARAMETER}

For c.m. energies larger than $5 \mathrm{TeV}$, Eq. 7 indicates a larger demagnification and a larger disruption are desirable. From the chromatic condition

$$
\left(\frac{\sigma^{*}}{\sigma_{1}}\right)^{2}=\left(\frac{\ell^{*}}{\ell_{1}}\right)^{2}+\frac{\ell^{*}}{\ell_{1}}\left(\frac{\ell^{*}}{\ell^{1}} \xi^{2}-2\right) \bar{\delta}^{2}+\bar{\delta}^{4}
$$

and for $\ell^{*} / \ell_{1}=400$, the demagnification $\xi$ can be as large as 30 and one can still have a $2 \%$ bandwidth. However, the tolerances on the lens beam quality become more stringent for larger demagnification.

\section{LENS-BEAM CURRENT}

The lens-beam parameters in Table 1 appear reasonable enough. However the round main-beam parameters have a small bunch charge and imply a train with many bunches.
Table 1: Parameters

$\begin{array}{lrrrr}\text { c.m. energy }[\mathrm{TeV}] & 1 / 2 & 1 & 11 / 2 & 3 \\ \gamma_{M} & 5 . \mathrm{E} 5 & 1 . \mathrm{E} 6 & 1.5 \mathrm{E} 6 & 3.0 \mathrm{E} 6 \\ \gamma_{Q}[\mu \mathrm{m}] & 5 . \mathrm{E} 3 & 9.9 \mathrm{E} 3 & 1.5 \mathrm{E} 4 & 3 . \mathrm{E} 4 \\ \sigma_{z, M}[\mu \mathrm{m}] & 182 & 129 & 70.2 & 82.8 \\ \sigma_{z, Q}[\mu \mathrm{m}] & 1170 & 824 & 449 & 529 \\ (\gamma \epsilon)_{M}[\mu \mathrm{m}] & .543 & .221 & .103 & .0733 \\ (\gamma \epsilon)_{Q}[\mu \mathrm{m}] & 2.88 & 1.17 & .544 & .389 \\ N_{M}\left[10^{10}\right] & .204 & .0829 & .0385 & .0276 \\ N_{Q}\left[10^{10}\right] & 2.44 & .989 & .460 & .329 \\ \ell^{*}[\mathrm{~cm}] & 3.54 & 2.50 & 1.36 & 1.61 \\ \delta_{B}[\%] & 2.3 & 5.3 & 10.4 & 13.8 \\ \Upsilon_{\text {avg }} & 0.06 & 0.19 & 0.59 & 1.10 \\ n_{B}(\mathrm{rep} \text { rate } 120 \mathrm{~Hz}) & 565 & 983 & 1727 & 1708 \\ \text { L }\left[10^{34} \mathrm{~cm}{ }^{-2} \text { sec }\right. & -1 \\ \text { Lens-main collision: } & 0.5 & 1.0 & 2.25 & 2.70 \\ \beta_{Q}^{*}[\mathrm{~mm}](\text { waist in front) } & .781 & .552 & .301 & .354 \\ \sigma_{M}[\mathrm{~nm}] & 1160 & 440 & 181 & 117 \\ \sigma_{Q}[\mathrm{~nm}] & 2010 & 762 & 313 & 203 \\ \text { Main-main collision: } & & & & \\ \sigma_{f}[\mathrm{~nm}] & 40.6 & 15.4 & 6.32 & 4.10 \\ \sigma_{0}[\mathrm{~nm}] & 33.2 & 12.6 & 5.16 & 3.35 \\ \beta^{*}[\mathrm{~mm}] & 1.01 & .716 & .389 & .459\end{array}$

To maintain the efficiency of the main linac it is necessary to maintain a beam current (during the pulse) of about 0.5 Amps. Remarkably this optimum does not depend much on the fundamental frequency of the accelerating structures. For the main-beam bunch charge at $1 \mathrm{TeV}$ the required spacing equals the C-band wavelength. Furthermore, since

$$
N_{Q T}=\frac{N_{Q T}}{N_{Q D}} \frac{\sigma_{Q}^{2}}{\sigma_{M}^{2}} N_{Q 0} \approx 3.6 N_{Q 0}
$$

we have

$$
\frac{N_{Q T}}{N_{M}}=\frac{\gamma_{M}}{\gamma_{Q}} \frac{P_{Q}}{P_{M}}=\frac{\gamma_{M}}{\gamma_{Q}} \frac{N_{Q T}}{N_{Q 0}} \frac{P_{Q 0}}{P_{M}} \approx 12 .
$$

This implies the lens-beam current would be about 6 Amps. This is a large current for a ring (the PEP LER is designed for 2 Amps) and implies a major perturbation to the rf system as the 84-meter bunch train passes. We have not decided on the best way to overcome this problem. One solution is to have a lower current in the damping ring and use a combiner ring to compress the bunch train much as in the two-beam accelerator drive beam complex. And since the beam is only in the combiner ring for a few revolutions, that ring is simple and need not have an rf system.

\section{LENS-BEAM PROPERTIES}

We have described two physical configurations of the lens beam beamlines: i) injection from a damping ring into a jitter-correction and beam-shaping loop into the IP region[2], and ii) injection from a damping ring into a linac 
followed by a jitter-correction and beam-shaping loop[3]. If one desires a lens-beam system that extends to $3 \mathrm{TeV}$ c.m., the pure damping ring solution becomes very difficult. For this reason, and because of a need for bunch compression, we presently favor the linac solution.

The lens beam must have the following systems and properties:

- an energy about 1/100th of the main beam energy,

- an emittance given by

$$
(\gamma \epsilon)_{Q} \leq \frac{1}{4} \frac{\beta_{Q}^{*}}{2 f_{Q}} \frac{\sigma_{Q}^{2}}{\sigma_{M}^{2}} N_{M} r_{e}
$$

which is about $1 \mu \mathrm{m}-\mathrm{r}$ at $1 \mathrm{TeV}$ c.m. and does not get too much smaller,

- a final bunch length of about $\sigma_{z, Q}=0.5 \mathrm{~mm}$,

- number of electrons per bunch $\leq 10^{10}$,

- a current entering the IP of about $6 \mathrm{Amps}$,

- bunch-to-bunch jitter at the IP of less than $1 \%$,

- a $\beta_{Q}^{*}$ that would be about $2 \mathrm{~mm}$ in the absence of a lens-lens collision and that is about $0.5 \mathrm{~mm}$ with the lens-lens collision,

- a transverse distribution approximating a uniform disk at the lens-main collision,

- recapture of positrons and reinjection into a damping ring with a transverse emittance that can be redamped to the design emittance in a few damping times.

To meet these requirements this system probably contains

- two damping rings at about $2 \mathrm{GeV}$,

- bunch compression systems upon extraction from the damping ring,

- combiner rings following the bunch compression, probably containing jitter damping systems,

- an efficient S-band or C-band linac for acceleration to final lens-beam energy,

- loops after the linac each containing octupole modules for beam shaping, and perhaps feed-forward jitter controls,

- chromatically-corrected incoming final-focus systems for an incoming $\beta_{Q}^{*}$ of about $2 \mathrm{~mm}$,

- crab cavities at the entrance to the detector region,

- recapture loops after the IP,

- a linear wiggler for reducing at least positrons to the damping ring energy,

- a reinjection loop into the damping rings.

This is not a simple beam system, and perhaps it will not represent a cost savings compared to the conventional system. However, a centralized campus for main damping rings and low-energy linacs would allow the lens-beam linac to double as a main-beam pre-linac (to $10 \mathrm{GeV}$ ), and the returning lens-beam electrons could be used to create positrons. Major cost reductions and improved operability should follow from the round beam parameters with their lower charge, the wider focusing bandwidth (2\%) that reduces energy compensation tolerances, the absence of a collimation system, and absence of final focusing elements in the detector. The system scales to energies of $3 \mathrm{TeV}$ c.m. and perhaps higher, and is self-aligning at $3 \mathrm{TeV}$ c.m. and below.

\section{DISCUSSION AND CONCLUSIONS}

Simulations of the entire series of beam manipulations, from shaping of the lens beam by octupoles, through the lens-lens collision, lens-main collisions, and main-main collisions are in progress. In Figure 1 we show an example of a main beam distribution, after GUINEAPIG simulation of the lens-main collision and then transport by $\ell^{*}=25 \mathrm{~mm}$ to the main-main interaction point. The full simulations have yet to be completed; of particular importance will be to demonstrate in detail that it is possible to get sufficiently uniform lenses at both the lens-lens and lens-main collisions. With the caveat that such simulations bear out our expectations, dynamic focusing appears to be a promising alternative to conventional final-focus and collimation systems. We note also that a first experiment[7] has shown that the crab cavity phases can be measured with the required precision of 0.01 degrees at X-band.

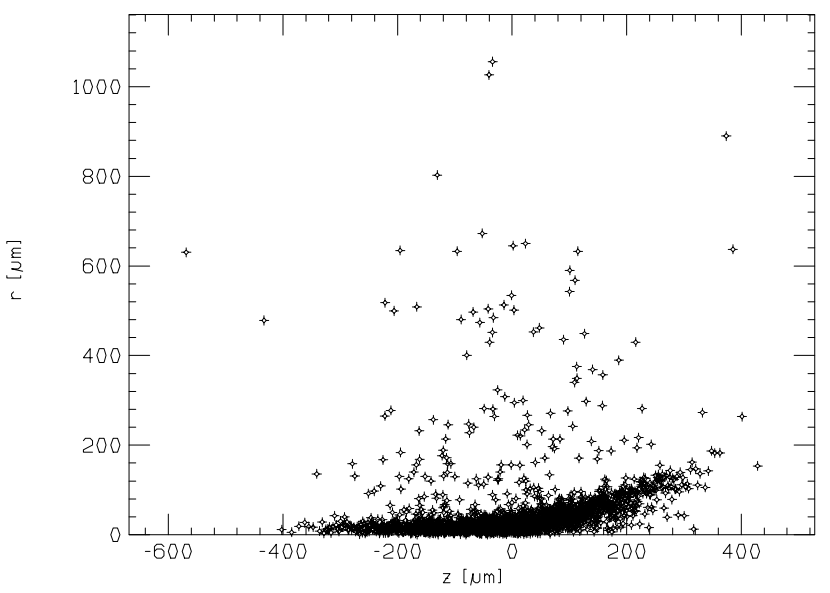

Figure 1: Main beam distribution after lens-main collision and transport by $\ell^{*}=25 \mathrm{~mm}$ to main-main collision.

\section{REFERENCES}

[1] J.Irwin, Proceedings IEEE Particle Accelerator Conference (PAC97), Vancouver, B.C., 12-16 May 1997.

[2] J.Irwin, 8th Workshop on Advanced Accelerator Concepts (AAC98), Baltimore, Maryland, 5-11 July 1998.

[3] J.Irwin, 19th International Linear Accelerator Conference (LINAC98), Chicago, 23-28 Aug 1998.

[4] D.Schulte, Ph.D. thesis (1996), TESLA-97-08.

[5] K. Oide, Phys.Rev.Lett., 61, 1713 (10 October 1988).

[6] Ref 2. Eq. 1 for pinch. There was a factor of 2 error in the original equation which is corrected here.

[7] S.Tantawi, private communication. 